\title{
Sparse Robust Filters for Scene Classification of Synthetic Aperture Radar (SAR) Images
}

\author{
Shuyuan Yang, Min Wang ${ }^{*}$, Hezhao Long, Zhi Liu \\ Key Lab of National Radar Signal Processing, School of Electronic Engineering, \\ Xidian University, Xi’an, Shaanxi, China, 710071 \\ Email: wangmin@xidian.edu.cn
}

\begin{abstract}
With the increasing resolution of Synthetic Aperture Radar (SAR) images, extracting their discriminative features for scenes classification has become a challenging task, because SAR images are very sensitive to target aspect brought by shadowing effects, interaction of the signature with the environment, and so on. Moreover, SAR images are remarkably polluted by the multiplicative speckle noise, which makes the conventional feature extractors inefficient. In this paper we advance new Sparse Robust Filters (SRFs) for automatic learning of discriminant features of scenes. A Hierarchical Group Sparse Coding (HGSC) model is proposed to learn a set of sparse and robust filters, to capture the multiscale local descriptors that are robust to noises. Some experiments are taken on a TerraSAR-X images dataset (in the middle of the Swabian Jura, the Nördlinger Ries, HH, observed on July, 2007), and a Moving and Stationary Target Acquisition and Recognition (MSTAR) dataset, to evaluate the performance of our proposed method. The experimental results show that our method can achieve higher classification accuracy compared with other related approaches.
\end{abstract}

Index Terms - Scene classification; synthetic aperture radar; sparse robust filters; hierarchical group sparse coding

\section{INTRODUCTION}

Synthetic Aperture Radar (SAR) imaging technology has made rapid developments in recent years. Enormous amount of high spatial SAR images are collected every day from the increasing number of launched imaging platforms, which contain much important information for the subsequent decision in both the military and civil applications. As a consequence, these huge volume of SAR data need a rapid and automatic understanding and interpretation by computers. For more efficient interpretation of SAR data, one often need to first recognize the semantic category of a scene and then discover the semantically meaningful information contained within scenes [1-5], for target detection, target segmentation, target recognition and so on. Consequently, scene classification has been one of the most fundamental tasks in SAR images understanding and interpretation [6-9].

Scene classification of SAR images is a challenging task because one scene often covers multiple land-cover classes or ground objects. Feature extraction is the first and most critical step in the scene classification, whose aim is to find a 
highly discriminative descriptor to predict the category of the scene. It can remarkably influence the classification results and the subsequent understanding of SAR images. Manually designing feature representations is very popular in the feature extraction of SAR images [9-18]. Early feature extractors of SAR images aim to develop a single unified model to jointly describe all aspects of the scene [9-10]. However, a scene can be described in many ways or from multiple views, including regions, geometric structures, textures, and so on [11-18]. Some texture features, such as gray-level co-occurrence matrix (GLCM) [1], Gabor filter [2], Gaussian Markov random field (GMRF) [3] and some complex structure in a local context [6], have been used for extracting discriminative features. Sparse coding is also adopted to encode the image scenes by a set of generated basis functions. Recently, researches on the description of SAR images have proceeded along many separate trajectories [19-23]. For example, Scale-Invariant-Feature-Transform (SIFT)-based feature descriptors and sparse coding $(\mathrm{SIFT}+\mathrm{SC})$ proved to output the state-of-the-art results. However, manually designing a combination of various types of features is a boring "feature engineering". Moreover, the feature extraction of SAR images is especially difficult, for the following reasons: 1) SAR images are corrupted by multiplicative speckle noises due to random interference of electromagnetic waves, and more land-covers or ground objects are contained in a scene compared with natural images; 2) SAR images are very sensitive to target aspect brought by shadowing effects, interaction of the signature with the environment, and so on; 3) The increasing resolution of SAR images makes the spatial and structural information in the image more complex. These characteristics of SAR images make the traditional low-level feature extractors (e.g., pixels, texture, and shapes) insufficient for extracting highly discriminative features and achieving satisfactory classification results.

Very recently one of the trends in image descriptors is to learn a high-level summary or representation of an image without explicitly describing the context within it. One of its realizations is the unsupervised feature learning, which has been shown to be effective at learning representations of images, videos and audios [24-37]. Many deep learning models, including Auto-Encoders (AEs) [28], Restricted Boltzmann Machines (RBMs) [38] et.al, have been developed for learning low- dimensional feature representations to reduce the data dimensionality. Although these approaches can avoid manually designing discriminative features, they are always challenging to implement for the requirement of tuning various parameters. For example, RBMs often has half a dozen parameters and an intractable objective function, which makes it hard to tune and converge [38-39].

In order to well understand computational principles or neural mechanisms of the feature extraction process, one should expand our view of biological underpinnings of visual sensing. Consequently, combing purely computational models with biological evidences is very promising for deriving a set of sophisticated features from complex scenes. Recently sparse representation or coding has been developed to explain early visual cortex as an efficient means of 
coding [40-43]. Sparse coding has found successful applications in machine learning, which can approximate data using a few non-zero coefficients (features) and provide insights into designing new algorithms based on optimizing for desirable properties of features [19-22]. For the characteristic of simple principle, robustness to noise, and flexible representation, sparse coding is potential to process complex and heavily polluted SAR images. By simulating the sparse coding mechanism of visual neuron, we can learn sets of over-complete bases to represent SAR data efficiently. Moreover, visual organization rules state that visual forms are not only sparse, but also hierarchical or deep. In recent years, the neocortex of the mammalian brain proves to organize in a complex layered structure of neurons. The past few years have witnessed prosperity of establishing deep architectures for modeling complex structured data [1-3]. Therefore, deep architectures are useful to solve complex cognition tasks, where the multiscale information of a complex scene can be captured via hierarchical learning.

In this paper, we combine the sparsity/selective characteristic and deep architectures of the visual organization rules together, and use these biological evidences to develop a simple and efficient sparse feature learning model to describe SAR image scenes. In our method, we cast a two-fold sparsity on the discriminative features, and extend the sparse coding to the multiscale case, to develop a Hierarchical Group Sparse Coding (HGSC) model for representing data and making it easier to extract useful information when building classifiers. The Treves-Rolls [44-45] measure is employed to characterize the sparsity of features, and a total variation regularizer is cast on the filters optimization to reduce the noise. Consequently, the proposed HGSC can not only exploit the discrimination between different classes of scenes, but also

robust to intrinsic speckle noises. Some experiments are taken on a TerraSAR-X images dataset (in the middle of the Swabian Jura, the Nördlinger Ries, HH, observed on July, 2007), and a Moving and Stationary Target Acquisition and Recognition (MSTAR) dataset, to evaluate the performance of our proposed method. The experimental results show that our proposed method can achieve higher classification accuracy when compared with other related approaches.

The rest of this paper is organized as follows. Section II introduces the proposed sparse robust filters. Section III reports the experimental results, with a comparison with some related feature extraction approaches. Conclusions are finally given in Section IV.

\section{Sparse Robust FiLters (SRFs) FOR ScENe ClassificAtion}

Given one SAR image being certain classes of a scene, we first partition it into some small $\sqrt{n} \times \sqrt{n}$ patches, and then convert it into an $n$-dimensional vector $\mathbf{x}_{\boldsymbol{i}}(\boldsymbol{i}=1, \ldots, N)$, where $N$ is the number of patches. Then the whole image can be 
described as a patch matrix $\mathbf{X}=\left[\mathbf{x}_{1}, \ldots, \mathbf{x}_{N}\right] \in \boldsymbol{R}^{n \times N}$, whose column is a patch or example. In our method, we first assume that the features of $\mathbf{X}$ are generated through some deterministic nonlinear transformation, that is, the $j-$ th feature of the sample $\mathbf{x}_{i}$ is calculated by $f_{i}^{j}=\phi\left(\mathbf{w}_{j}^{T} \mathbf{x}_{i}\right)(i=1, \ldots, N ; j=1, \ldots, d)$, where $\phi(\cdot)$ is a nonlinear transformation function and $\left\{\mathbf{w}_{j}\right\}_{j=1}^{d}$ are a set of projection functions or filters to generate features. Combing the features $f_{i}^{j}$ together, we can derive a feature matrix $\mathbf{F}$,

$$
\mathbf{F}=\phi(\mathbf{W X})=\left[\mathbf{f}_{1}, \ldots, \mathbf{f}_{N}\right]=\left[\left(\mathbf{f}^{1}\right)^{T}, \ldots .,\left(\mathbf{f}^{d}\right)^{T}\right]^{T}
$$

where $\mathbf{W}=\left[\mathbf{w}_{1}, \ldots, \mathbf{w}_{\boldsymbol{d}}\right] \in \boldsymbol{R}^{\boldsymbol{d} \times \boldsymbol{n}}$ and $\mathbf{F} \in \boldsymbol{R}^{\boldsymbol{d} \times \boldsymbol{N}}$ in the formula (1). In the feature matrix $\mathbf{F}$, each entry $f_{i}^{j}$ is the activity of feature $j$ on the $i$-th example. Each column in $\mathbf{F}$ is denoted as $\mathbf{f}_{i} \in \boldsymbol{R}^{d}(\boldsymbol{i}=1, \ldots, \boldsymbol{N})$ ( $d$ is the number of features) and each row is denoted as $\mathbf{f}^{j} \in \boldsymbol{R}^{N}(\boldsymbol{j}=1, \ldots, \boldsymbol{d})$. As mentioned in the introduction, a computational feature learning model should not only be descriptive, mathematical, algorithmic/computational about the feature calculation process, but also attempt to mimic and explain visual attentive behaviors, such as the sparse characteristic and deep architectures of the visual organization rules. In the following some desirable properties of the feature matrix will be discussed.

\section{A. Two-fold Sparsity of Features}

The neuron activations in the human brain have the characteristic of sparsity. First, only limited number of neurons are active when one sees a scene. Second, for a given neuron, it does not response to the whole scene but a local region. In our method, this two-fold sparsity of the feature matrix is explored, which can be summarized as the sparsity along the rows and columns respectively. Concretely, only a small number of features of one sample are remarkable while others are not, that is, each $\mathbf{f}_{i}$ in the feature matrix $\mathbf{F}$ should be sparse. On the other hand, in order to keep the discrimination ability of features, only a small number of examples are active when receiving multiple examples, that is, each $\mathbf{f}^{j}$ in the feature matrix $\mathbf{F}$ should be sparse. In our work, the feature matrix is considered to have this two-fold sparsity.

Moreover, our method casts an assumption that each feature is equally active on the feature matrix $\mathbf{F}$, via dividing each feature by its 2-norm across all examples, that is, $\left\|\mathbf{f}^{j}\right\|_{2}=1$. Each sample is considered to have unit length, i.e., $\left\|\mathbf{f}_{i}\right\|_{2}=1$. Consequently, finding the features with two-fold sparsity can be reduced to the following optimization problem,

$$
\left\{\begin{array}{c}
\underset{\mathbf{f}}{\min } \sum_{\boldsymbol{i}}\left\|\mathbf{f}_{\boldsymbol{i}}\right\|_{0}+\sum_{\boldsymbol{j}}\left\|^{\boldsymbol{j}}\right\|_{0} \\
\boldsymbol{s . t}\left\|\mathbf{f}_{\boldsymbol{i}}\right\|_{2}=1 ;\left\|\mathbf{f}^{\boldsymbol{j}}\right\|_{2}=1
\end{array}\right.
$$

Replacing $\mathbf{F}=\phi(\mathbf{W X})$ into the formula (2), we can reformulate the formula (2) as, 


$$
\left\{\begin{array}{l}
\min _{\mathbf{w}}\|\phi(\mathbf{W X})\|_{\text {row }, 0}+\|\phi(\mathbf{W X})\|_{\text {col, } 0} \\
\text { s.t }\left\|\phi\left(\mathbf{W} \mathbf{x}_{i}\right)\right\|_{2}=1 ; \quad\left\|\phi\left(\mathbf{w}_{\boldsymbol{i}} \mathbf{X}\right)\right\|_{2}=1, \quad \forall \mathbf{i}
\end{array}\right.
$$

It is a Group Sparse Coding (GSC) model for representing data and extracting useful information. As soon as the solution to the formula (3) is obtained, we can obtain a group of sparse filters $\left\{\mathbf{w}_{i}\right\}$ from $\mathbf{W}$.

\section{B. Hierarchical Sparse and Robust Filters}

Because SAR images are often heavily polluted by speckle noises, the learned filters cannot reveal the low-level features such as edges, contours and texons. In our work we develop a set of sparse and robust filters, to reduce the influence of intrinsic noises on the feature extraction. The noises in the image would generate some unwanted features and degrade the classification results. So in our method, a total variation regularizer is cast on the filters optimization to make them robust to noises. That is, we hope the derivation of $\phi(\mathbf{W X})$ can be minimized, which can be written as, $\sum_{i}\left\|\nabla \phi\left(\mathrm{W} \mathbf{x}_{i}\right)\right\|_{1}$. The optimization of the L1 norm of gradients will reduce the unwanted singular features, which is some like a nonlinear denoising filtering. Introducing this regularizer into the GSC model in the formula (3), we can obtain,

$$
\left\{\begin{array}{l}
\min _{\mathbf{w}}\left(\|\phi(\mathbf{W} \boldsymbol{X})\|_{\text {row }, 0}+\|\phi(\mathbf{W} \boldsymbol{X})\|_{\text {col, }, 0}\right)+\lambda\|\nabla \phi(\mathbf{W} \boldsymbol{X})\|_{1} \\
\text { s.t }\left\|\phi\left(\mathbf{W} \mathbf{x}_{i}\right)\right\|_{2}=1 ; \quad\left\|\phi\left(\mathbf{w}_{\boldsymbol{i}} \mathbf{X}\right)\right\|_{2}=1 \quad, \quad \forall \mathbf{i}
\end{array}\right.
$$

In this paper, an Alternating Direction Method of Multipliers (ADMM) algorithm [52] is advanced to solve (4). By introducing a new variable $\mathbf{H}$, the formula (4) is first changed to,

$$
\min _{\mathbf{W}, \mathbf{H}}\left(\|\phi(\mathbf{W} \boldsymbol{X})\|_{\text {row }, 0}+\|\phi(\mathbf{W} \boldsymbol{X})\|_{\text {col }, 0}\right)+\lambda\|\nabla \phi(\mathbf{H} \boldsymbol{X})\|_{1}+\sum_{i}\left[\left\|\phi\left(\mathbf{W} \mathbf{x}_{i}\right)\right\|_{2}-1\right]^{2}+\sum_{i}\left[\left\|\phi\left(\mathbf{w}_{i} \mathbf{X}\right)\right\|_{2}-1\right]^{2}+\|\mathbf{W}-\mathbf{H}\|^{2}
$$

The optimization problem is divided into two sub-problems: the optimization of $\mathbf{W}$ and $\mathbf{H}$.

In order to solve (5), firstly we fix $\mathbf{H}$ and optimize $\mathbf{W}$ by solving (6),

$$
\min _{\mathbf{W}}\left(\|\phi(\mathbf{W} \mathbf{X})\|_{\text {row }, 0}+\|\phi(\mathbf{W} \mathbf{X})\|_{\text {col, } 0}\right)+\sum_{i}\left[\left\|\phi\left(\mathbf{W} \mathbf{x}_{i}\right)\right\|_{2}-1\right]^{2}+\sum_{i}\left[\left\|\phi\left(\mathbf{w}_{i} \mathbf{X}\right)\right\|_{2}-1\right]^{2}+\|\mathbf{W}-\mathbf{H}\|^{2}
$$

It can be achieved using the canonical greedy layerwise approach [46-47]. Then we fix $\mathbf{W}$ and optimize $\mathbf{H}$ by solving [53],

$$
\min _{\mathbf{H}} \lambda\|\nabla \phi(\mathbf{H X})\|_{1}+\|\mathbf{W}-\mathbf{H}\|^{2}
$$

The two variables update iteratively to derive a solution to (4), then we can obtain a group of sparse robust filters $\mathbf{W}^{1}, \mathbf{W}^{2}, \ldots$ Moreover, this process is cascaded to develop a Hierarchical Group Sparse Coding (HGSC) model for hierarchically representing patches, as shown in fig.1. When the features are extracted, they are processed by a maximal pooling to input into the next layer. 


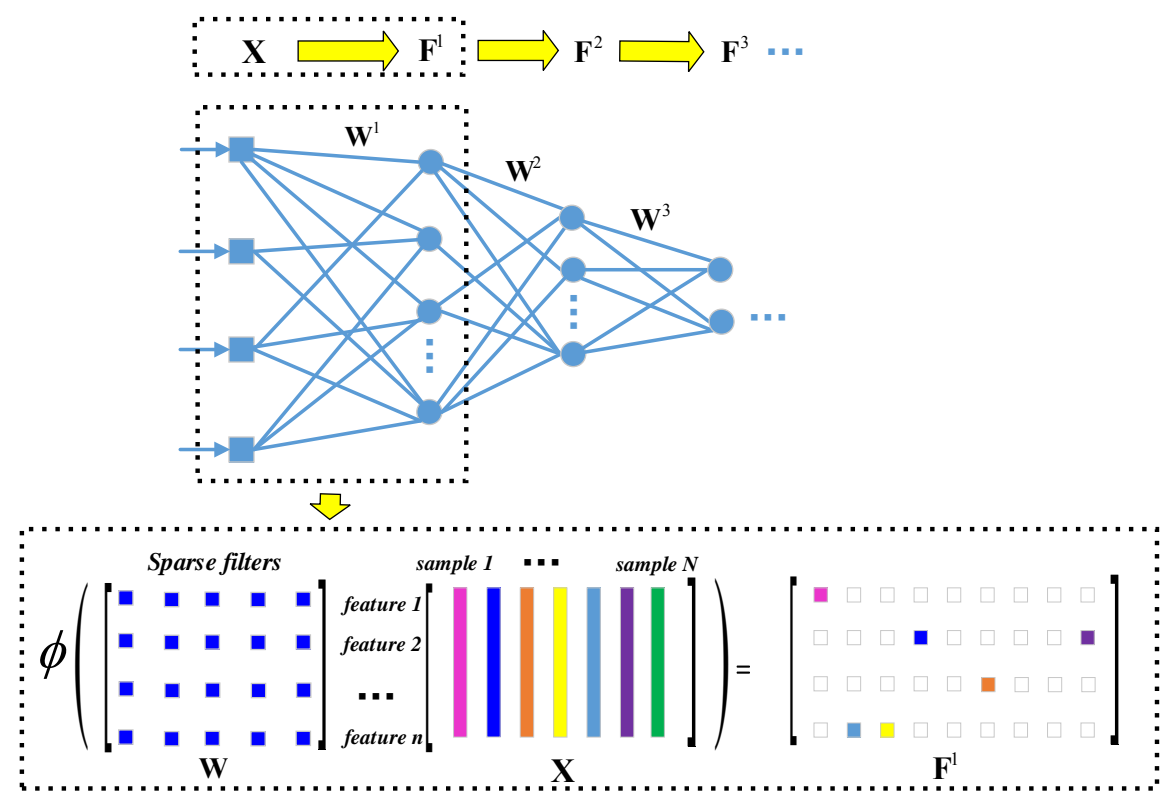

Fig.1 Generation of Hierarchical Sparse and Robust Filters via HGSC

\section{EXPERIMENTAL RESULTS AND DISCUSSIONS}

In this section, some experiments are taken to investigate the performance of our proposed method. Some experiments are carried to evaluate our method and compare it with some related approaches in the literature, including: 1) Principal Component Analysis (PCA)[7]; 2) Sparse Coding based feature extraction (SC) [20]; 3) Contextual Classification Algorithm (CCA) [22]; 4) CRFs [23]; 5) AE [28]; 6) RBM [38]. In the comparative deep networks, both AE and RBM can present the best results when $8 * 8$ patches are used, so in our method we choose $n=64$ for a fair comparison, and the number of patches $N=(256 * 256) /(8 * 8)=1024$. Three layers are used for feature extraction and a linear SVM is used for the classification, $\phi(x)=\sqrt{\left(\varepsilon+x^{2}\right)}, \varepsilon=0.1$. In this section, we applied sparse filtering to learn features from 5000 patches that are randomly sampled from the SAR images in two datasets. In SVM, C is chosen by hold-out cross-validation. For each image size, we learn a complete set of features (i.e., equal to the number of input dimensions). In the comparative methods, we set the layers of $\mathrm{AE}$ and $\mathrm{RBM}$ networks as three, and the number of filters in these two networks is also the same with that of our method. AE is implemented as described in Coates et al. [49]. In the sparse coding method, we used code from [50] for determining the coefficients. In CCA and CRFs, we use the parameters suggested in the literatures. The simulations of different methods are carried out in MATLAB 7.10.0(R2010a) environment running in Core 2 Quad, 2.99-GHZ CPU with 2-GB RAM. 30 independent experiments are undertaken, and the average results are calculated for a comparison. 


\section{A. Datasets}

Dataset 1: The performance of our proposed method is first tested on a TerraSAR-X images database containing 10600 scene images (in the middle of the Swabian Jura, the Nördlinger Ries, HH, observed on July, 2007). In the dataset there are six classes of scenes, including city, airport, farmland, bridge, mountain, and water, some samples are shown in fig.2. All the images in this dataset are of size $256 \times 256$.
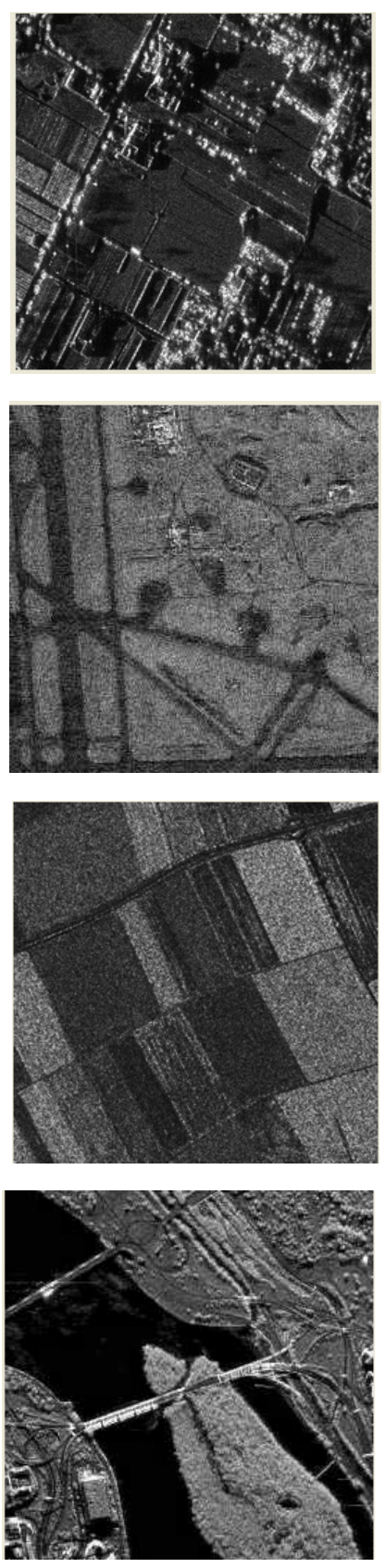

(d) bridge
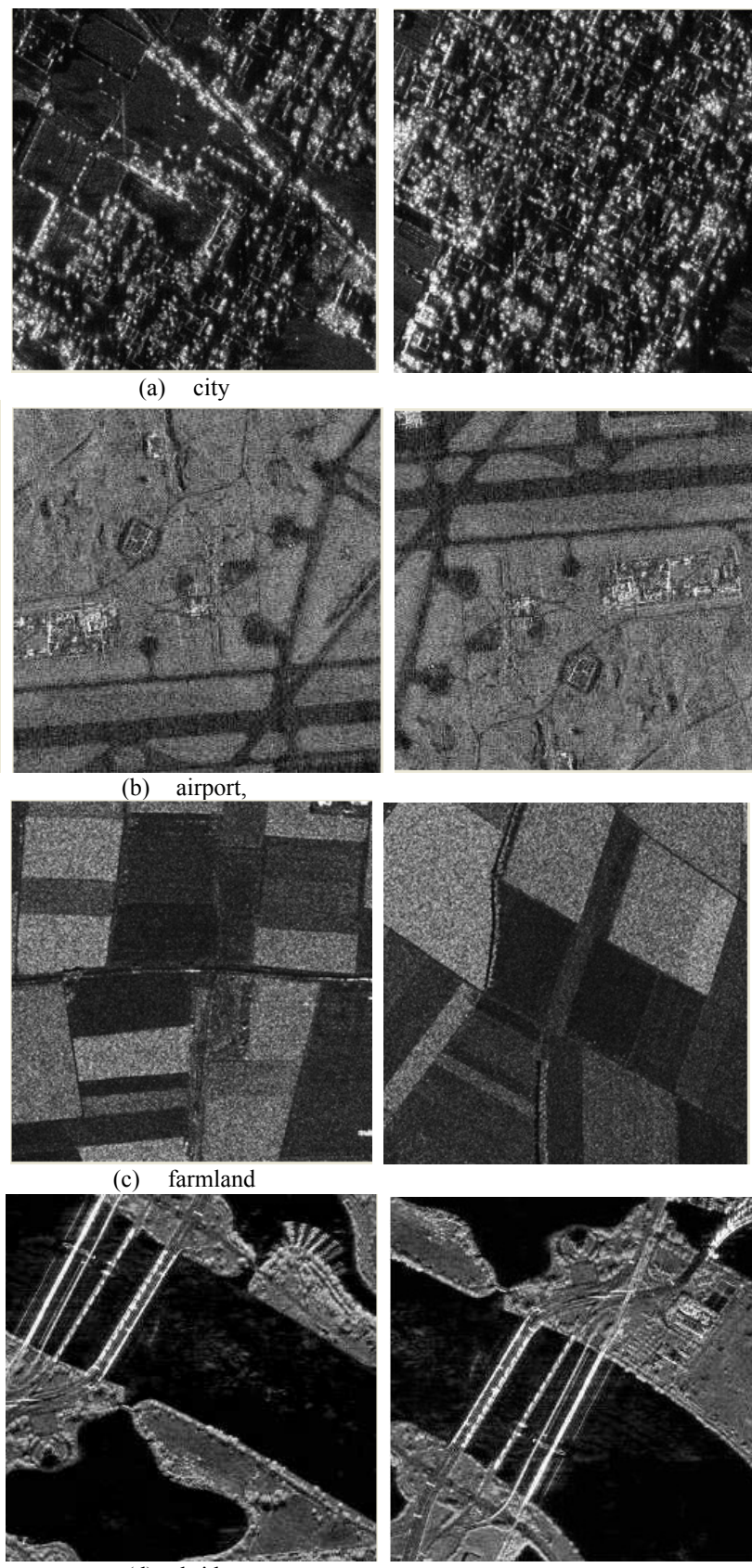

(a) city
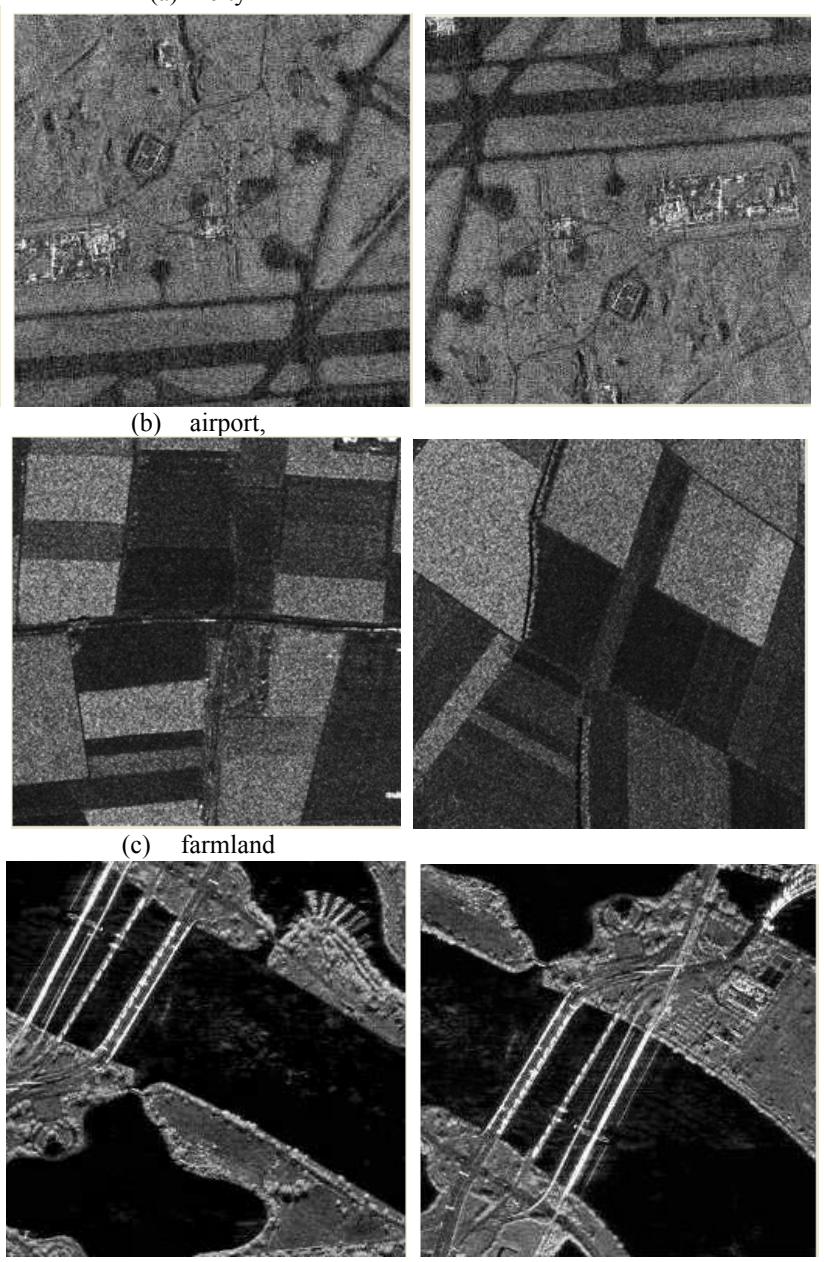

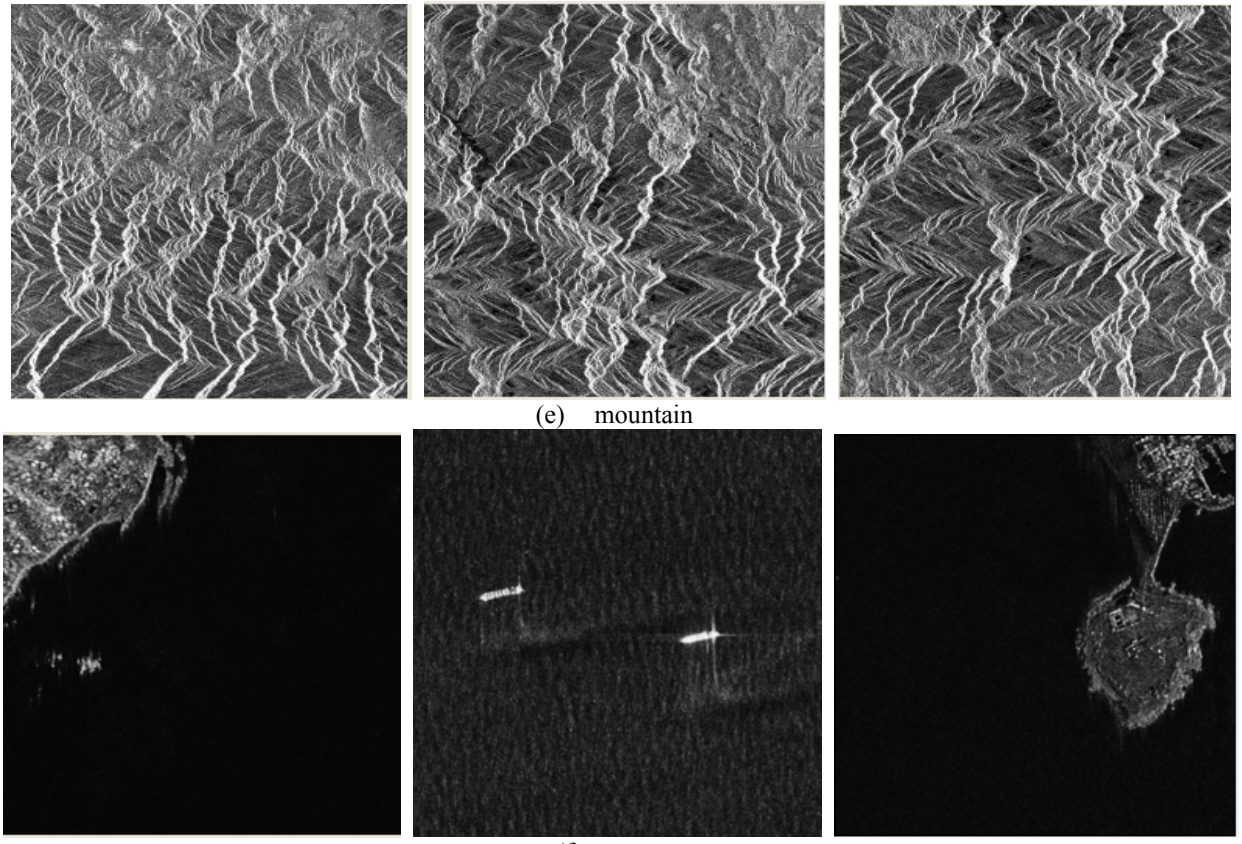

(f) water

Fig.2 Some example samples in the database.

Dataset 2: The second dataset is a Moving and Stationary Target Acquisition and Recognition (MSTAR) dataset. These SAR images are collected in spotlight mode at $0.3 m \times 0.3 m$ resolution with HH polarization by the Sandia National Laboratory X-band SAR sensor. And it contains 10 classes of military vehicles. Each of the targets has views at $15^{\circ}$ and $17^{\circ}$ depression angles [51][54]. In our experiments, we use three types of vehicles, including BMP2 (tank), BTR70 (armored car) and T72 (tank), to evaluate the performance of our proposed scheme. The data in depression $17^{\circ}$ are used for training and the rest for testing. The number of training and test samples of each target is shown in Table I.

TABLE I

THE NUMBER OF TRAINING AND TESTING SAMPLES FOR EACH TYPE OF TARGET

\begin{tabular}{|c|c|c|c|c|c|}
\hline Vehicle $\left(17^{\circ}\right)$ & \#Training Samples & \#Testing Samples & $\operatorname{Vehicle}\left(17^{\circ}\right)$ & \#Training Samples & \#Testing Samples \\
\hline BMP2(S-C9563) & 233 & 195 & BMP2(S-C 21) & 233 & 196 \\
\hline BMP2(S-C 9566) & 232 & 196 & BTR70(SN-C71) & 233 & 196 \\
\hline BMP2(S-C 21) & 233 & 196 & $\mathrm{~T} 72(\mathrm{SN}-132)$ & 232 & 196 \\
\hline BTR70(SN-C71) & 233 & 196 & $\mathrm{~T} 72(\mathrm{SN}-812)$ & 231 & 195 \\
\hline $\mathrm{T} 72(\mathrm{SN}-132)$ & 232 & 196 & T72(SN-S7) & 228 & 191 \\
\hline $\mathrm{T} 72(\mathrm{SN}-812)$ & 231 & 195 & BMP2(S-C 9566) & 232 & 196 \\
\hline $\mathrm{T} 72(\mathrm{SN}-\mathrm{S} 7)$ & 228 & 191 & BMP2(S-C 21) & 233 & 196 \\
\hline BMP2(S-C 9566) & 232 & 196 & & & \\
\hline
\end{tabular}

Some example training images (including nine images in dataset 1 and three types of vehicles' images in dataset 2) are shown in Fig.3. 

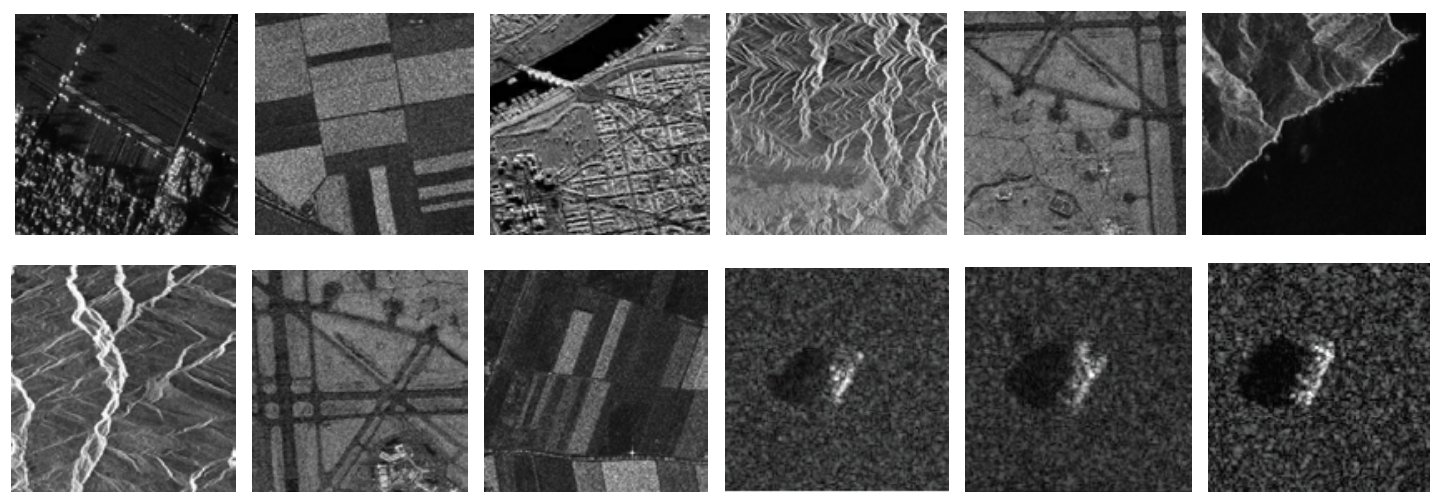

Fig.3 Training examples in dataset 1 and dataset 2

\section{Experiment 1: Classification results on Dataset 1}

In this test, we classify this dataset by different methods and change the number of training samples. The training samples of each class take 10, 20, 40, 60 respectively, and the number of the total training samples is $60,120,240$ and 360 respectively. The experiments are taken under the condition in section III.A. The classification accuracy of PCA[7], $\mathrm{SC}[20], \mathrm{CCA}[22], \mathrm{CRFs}[23], \mathrm{AE}[28], \mathrm{RBM}$ [38] and our method are shown in table II. From it we can see that for the seven methods, the classification accuracy increases with the number of training samples. Compared with the shallow models such as the PCA[7], SC[20], $\mathrm{CCA}[22]$ and $\mathrm{CRFs}[23]$, the hierarchical models (including AE, RBM and our method) can obtain higher recognition accuracy, for exploring the deep properties of organization in a complex layered structure of neurons. Moreover, our method can achieve comparable classification accuracy with the two deep models when there are small number of training samples. With the increase of the number of training samples, our method can obtain accurate classification results than the two deep networks, for learning a set of sparse and robust filters, to capture the multiscale local descriptors that are robust to noises.

\section{TABLE II}

The recognition accuracy (\%) for different approaches

\begin{tabular}{ccccccccc}
\hline \hline \#training samples & PCA[7] & SC[20] & CCA[22] & CRFs[23] & AE[28] & RBM[38] & Our method \\
60 & 70.22 & 71.90 & 73.04 & 72.32 & 78.47 & $\mathbf{8 0 . 3 3}$ & 79.48 \\
120 & 71.24 & 74.39 & 75.88 & 74.90 & 82.11 & 83.89 & $\mathbf{8 3 . 2 7}$ \\
240 & 74.27 & 76.02 & 77.36 & 75.25 & 84.49 & 89.92 & $\mathbf{9 0 . 4 1}$ \\
360 & 76.33 & 79.47 & 80.04 & 78.93 & 86.52 & 90.26 & $\mathbf{9 2 . 3 5}$ \\
\hline \hline
\end{tabular}

\section{B. Experiment 2: Classification results on Dataset 2}

In this experiment, we classify the dataset 2 by using different methods (including PCA[7], SC[20], CCA[22], CRFs[23], AE[28], RBM[38] and our method) under the condition described in section III.A. For all the methods, 100 images per class are taken from the dataset and used as training samples. Fig.4 plots the confusion matrix of the classification result by our method. From fig. 4 we can see that our method can achieve accurate recognition for the seven 
types of objects.

\begin{tabular}{|c|c|c|c|c|c|c|}
\hline AP2(S-C9563 & P2(S-C 9566) & BMP2(S-C 21) & BTR70(SN-C71) & $\mathrm{T} 72(\mathrm{SN}-132)$ & T72(SN-812) & $\mathrm{T} 72(\mathrm{SN}-\mathrm{S} 7)$ \\
\hline 183.00 & 4.00 & 4.00 & 0.00 & 2.00 & 1.00 & 1.00 \\
\hline 3.00 & 185.00 & 5.00 & 1.00 & 0.00 & 2.00 & 0.00 \\
\hline 3.00 & 2.00 & 189.00 & 0.00 & 1.00 & 1.00 & 0.00 \\
\hline 1.00 & 3.00 & 1.00 & 189.00 & 0.00 & 1.00 & 1.00 \\
\hline 0.00 & 1.00 & 2.00 & 0.00 & 190.00 & 2.00 & 1.00 \\
\hline 0.00 & 1.00 & 1.00 & 0.00 & 2.00 & 188.00 & 3.00 \\
\hline 0.00 & 1.00 & 0.00 & 0.00 & 2.00 & 2.00 & 186.00 \\
\hline
\end{tabular}

Fig.4 The confusion matrix of the classification result by our method

Moreover, we compare the classification results of PCA[7], SC[20], CCA[22], CRFs[23], AE[28], RBM[38] with our proposed method, and the results are shown in fig.5. From it we can see that our method can achieve more accurate classification than both the shallow models and two deep networks. Moreover, the deep models outperform the other methods remarkably for employing hierarchical model. In our method, SRFs can automatically learn the discriminant, features of scenes that are robust to noise, by simulating the sparse coding and hierarchical mechanism of visual neuron. Moreover, by adding a regularizer in filters learning, our method can present better results than the other two deep networks.

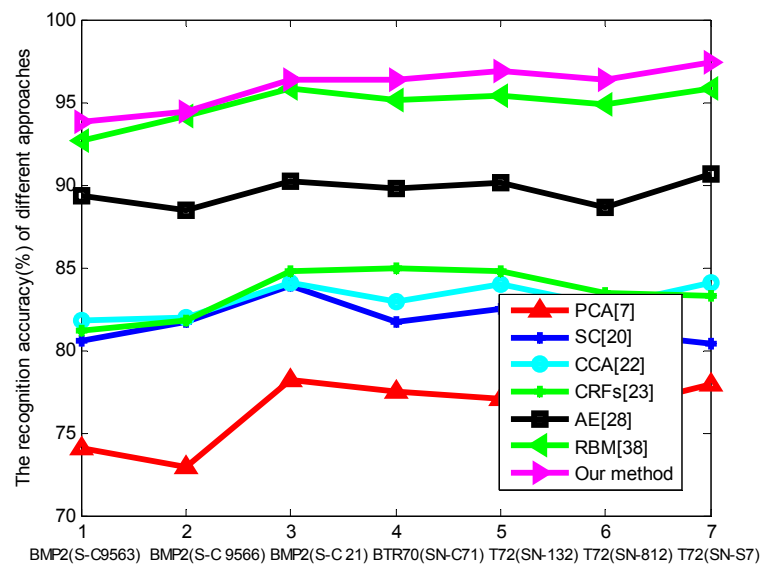

Fig.5 The comparative results of different methods

\section{CONCLUSIONS}

Inspired by the recently developed sparse coding, in this paper a new Sparse Robust Filters (SRF) based feature extraction approach is proposed. Hierarchical sparse representation is utilized to extract the discriminant features of 
scenes, and sparse and robust filters are designed to capture the multiscale local descriptors robust to noise. Some experiments are taken on a TerraSAR-X images dataset and a MSTAR dataset, to evaluate the performance of our proposed method. The experimental results show that our proposed method can achieve higher recognition accuracy compared with other related approaches.

\section{ACKNOWLEDGEMENTS}

The authors would like to thank the anonymous reviewers for their very constructive comments. This work was supported by the National Basic Research Program of China (973 Program) under Grant no.2013CB329402, the fundamental research funds for the central universities under grant no. BDY021429, Major Research Plan of the National Natural Science Foundation of China (no.91438103, 91438201), Huawei Innovation Research Program, the Kunshan innovation institute of Xidian University, NSFC 61072108, 61173090, NCET-10-668, the Foreign Scholars in University Research and Teaching Programs (No.B07048) and 2013KJXX-63.

\section{REFERENCES}

[1]. Pons Bernad, G.; Denise, L.; Refregier, P., "Hierarchical Feature-Based Classification Approach for Fast and User-Interactive SAR Image Interpretation," Geoscience and Remote Sensing Letters, IEEE, vol.6, no.1, pp.117,121, Jan. 2009

[2]. Tupin, F.; Bloch, I.; Maitre, H., "A first step toward automatic interpretation of SAR images using evidential fusion of several structure detectors," Geoscience and Remote Sensing, IEEE Transactions on , vol.37, no.3, pp.1327,1343, May 1999

[3]. Guida, R.; Iodice, Antonio; Riccio, Daniele; Stilla, U., "Model-Based Interpretation of High-Resolution SAR Images of Buildings," Selected Topics in Applied Earth Observations and Remote Sensing, IEEE Journal of , vol.1, no.2, pp.107,119, June 2008

[4]. Boerner, Wolfgang-M; Bing-yuen Foo; Eom, Hyo J., "Interpretation of the Polarimetric Co-Polarization Phase Tern in Radar Images Obtained with the JPL Airborne L-Band SAR System," Geoscience and Remote Sensing, IEEE Transactions on , vol.GE-25, no.1, pp.77,82, Jan. 1987

[5]. Saville, M.A.; Jackson, J.A.; Fuller, D.F., "Rethinking vehicle classification with wide-angle polarimetric SAR," Aerospace and Electronic Systems Magazine, IEEE , vol.29, no.1, pp.41,49, Jan. 2014 
[6]. Yongke Ding; Lizhong Qiu; Pinglv Yang; Zeming Zhou; Yuanxiang Li; Wenxian Yu, "Scene scattering descriptor for urban classification in very high resolution SAR images," Geoscience and Remote Sensing Symposium (IGARSS), 2013 IEEE International , vol., no., pp.2015,2018, 21-26 July 2013

[7]. Ferro-Famil, L.; Pottier, E.; Lee, J.S., "Unsupervised classification and analysis of natural scenes from polarimetric interferometric SAR data," Geoscience and Remote Sensing Symposium, 2001. IGARSS '01. IEEE 2001 International , vol.6, no., pp.2715,2717 vol.6, 2001

[8]. Yongfeng Cao; Junying Ren; Caixia Su; Jianjuan Liang, "Scene classification from PolSAR image using medium-level features," Synthetic Aperture Radar (APSAR), 2013 Asia-Pacific Conference on, vol., no., pp.342,345, 23-27 Sept. 2013

[9]. Fukuda, S.; Hirosawa, H., "A wavelet-based texture feature set applied to classification of multifrequency polarimetric SAR images," Geoscience and Remote Sensing, IEEE Transactions on, vol.37, no.5, pp.2282,2286, Sep 1999

[10]. Yin, H.; Cao, Y.; SUN, H., "Combining pyramid representation and AdaBoost for urban scene classification using high-resolution synthetic aperture radar images," Radar, Sonar \& Navigation, IET, vol.5, no.1, pp.58,64, January 2011

[11]. Deliang Xiang; Tao Tang; Lingjun Zhao; Yi Su, "Superpixel Generating Algorithm Based on Pixel Intensity and Location Similarity for SAR Image Classification," Geoscience and Remote Sensing Letters, IEEE , vol.10, no.6, pp.1414,1418, Nov. 2013

[12]. Maghsoudi, Y.; Collins, M.J.; Leckie, D.G., "Radarsat-2 Polarimetric SAR Data for Boreal Forest Classification Using SVM and a Wrapper Feature Selector," Selected Topics in Applied Earth Observations and Remote Sensing, IEEE Journal of , vol.6, no.3, pp.1531,1538, June 2013

[13]. Aytekin, O.; Koc, M.; Ulusoy, I., "Local Primitive Pattern for the Classification of SAR Images," Geoscience and Remote Sensing, IEEE Transactions on, vol.51, no.4, pp.2431,2441, April 2013

[14]. Voisin, A.; Krylov, V.A.; Moser, G.; Serpico, S.B.; Zerubia, J., "Classification of Very High Resolution SAR Images of Urban Areas Using Copulas and Texture in a Hierarchical Markov Random Field Model," Geoscience and Remote Sensing Letters, IEEE, vol.10, no.1, pp.96,100, Jan. 2013

[15].He, C.; Zhuo, T.; Zhao, S.; Yin, S.; Chen, D., "Particle Filter Sample Texton Feature for SAR Image Classification," Geoscience and Remote Sensing Letters, IEEE , vol.PP, no.99, pp.1,5 
[16]. Tao, M.; Zhou, F.; Liu, Y.; Zhang, Z., "Tensorial Independent Component Analysis-Based Feature Extraction for Polarimetric SAR Data Classification," Geoscience and Remote Sensing, IEEE Transactions on, vol.53, no.5, pp.2481,2495, May 2015

[17]. D'Elia, C.; Ruscino, S.; Abbate, M.; Aiazzi, B.; Baronti, S.; Alparone, L., "SAR Image Classification Through Information-Theoretic Textural Features, MRF Segmentation, and Object-Oriented Learning Vector Quantization," Selected Topics in Applied Earth Observations and Remote Sensing, IEEE Journal of, vol.7, no.4, pp.1116,1126, April 2014

[18]. Uhlmann, S.; Kiranyaz, S., "Integrating Color Features in Polarimetric SAR Image Classification," Geoscience and Remote Sensing, IEEE Transactions on , vol.52, no.4, pp.2197,2216, April 2014

[19]. Zhang, H.; Lin, H.; Li, Y., "Impacts of Feature Normalization on Optical and SAR Data Fusion for Land Use/Land Cover Classification," Geoscience and Remote Sensing Letters, IEEE , vol.PP, no.99, pp.1,5

[20]. Zhang, L.; Sun, L.; Zou, B.; Moon, W., "Fully Polarimetric SAR Image Classification via Sparse Representation and Polarimetric Features," Selected Topics in Applied Earth Observations and Remote Sensing, IEEE Journal of, vol.PP, no.99, pp.1,10

[21]. Banerjee, B.; Bhattacharya, A.; Buddhiraju, K.M., "A Generic Land-Cover Classification Framework for Polarimetric SAR Images Using the Optimum Touzi Decomposition Parameter Subset—An Insight on Mutual Information-Based Feature Selection Techniques," Selected Topics in Applied Earth Observations and Remote Sensing, IEEE Journal of , vol.7, no.4, pp.1167,1176, April 2014

[22]. Xin Niu; Yifang Ban, "A Novel Contextual Classification Algorithm for Multitemporal Polarimetric SAR Data," Geoscience and Remote Sensing Letters, IEEE , vol.11, no.3, pp.681,685, March 2014

[23]. Yongke Ding; Yuanxiang Li; Wenxian Yu, "SAR Image Classification Based on CRFs With Integration of Local Label Context and Pairwise Label Compatibility," Selected Topics in Applied Earth Observations and Remote Sensing, IEEE Journal of , vol.7, no.1, pp.300,306, Jan. 2014

[24]. Fan Zhang; Bo Du; Liangpei Zhang, "Saliency-Guided Unsupervised Feature Learning for Scene Classification," Geoscience and Remote Sensing, IEEE Transactions on , vol.53, no.4, pp.2175,2184, April 2015

[25]. Zhenyu Guo; Wang, Z.J., "An Unsupervised Hierarchical Feature Learning Framework for One-Shot Image Recognition," Multimedia, IEEE Transactions on , vol.15, no.3, pp.621,632, April 2013

[26]. Ling Shao; Li Liu; Xuelong Li, "Feature Learning for Image Classification Via Multiobjective Genetic Programming," Neural Networks and Learning Systems, IEEE Transactions on, vol.25, no.7, pp.1359,1371, July 2014 
[27]. Zhaoquan Yuan; Jitao Sang; Changsheng Xu; Yan Liu, "A Unified Framework of Latent Feature Learning in Social Media," Multimedia, IEEE Transactions on , vol.16, no.6, pp.1624,1635, Oct. 2014

[28]. Hoo-Chang Shin; Orton, M.R.; Collins, D.J.; Doran, S.J.; Leach, M.O., "Stacked Autoencoders for Unsupervised Feature Learning and Multiple Organ Detection in a Pilot Study Using 4D Patient Data,"Pattern Analysis and Machine Intelligence, IEEE Transactions on , vol.35, no.8, pp.1930,1943, Aug. 2013

[29]. Shiyang Lu; Zhiyong Wang; Tao Mei; Genliang Guan; Feng, D.D., "A Bag-of-Importance Model With Locality-Constrained Coding Based Feature Learning for Video Summarization," Multimedia, IEEE Transactions on , vol.16, no.6, pp.1497,1509, Oct. 2014

[30]. Zhen Zuo; Gang Wang, "Learning Discriminative Hierarchical Features for Object Recognition," Signal Processing Letters, IEEE , vol.21, no.9, pp.1159,1163, Sept. 2014

[31]. Jun Li; Xin Huang; Gamba, P.; Bioucas-Dias, J.M.; Liangpei Zhang; Atli Benediktsson, J.; Plaza, A., "Multiple Feature Learning for Hyperspectral Image Classification," Geoscience and Remote Sensing, IEEE Transactions on , vol.53, no.3, pp.1592,1606, March 2015

[32]. Yan, H.; Lu, J.; Zhou, X., "Prototype-Based Discriminative Feature Learning for Kinship Verification," Cybernetics, IEEE Transactions on , vol.PP, no.99, pp.1,1

[33]. Zhouyu Fu; Robles-Kelly, A., "Discriminant Absorption-Feature Learning for Material Classification," Geoscience and Remote Sensing, IEEE Transactions on , vol.49, no.5, pp.1536,1556, May 2011

[34]. Shuhui Bu; Zhenbao Liu; Junwei Han; Jun Wu; Rongrong Ji, "Learning High-Level Feature by Deep Belief Networks for 3-D Model Retrieval and Recognition," Multimedia, IEEE Transactions on, vol.16, no.8, pp.2154,2167, Dec. 2014

[35]. Han, J.; Zhang, D.; Cheng, G.; Guo, L.; Ren, J., "Object Detection in Optical Remote Sensing Images Based on Weakly Supervised Learning and High-Level Feature Learning," Geoscience and Remote Sensing, IEEE Transactions on , vol.PP, no.99, pp.1,13

[36]. Goodfellow, I.J.; Courville, A.; Bengio, Y., "Scaling Up Spike-and-Slab Models for Unsupervised Feature Learning," Pattern Analysis and Machine Intelligence, IEEE Transactions on, vol.35, no.8, pp.1902,1914, Aug. 2013

[37]. Farabet, C.; Couprie, C.; Najman, L.; LeCun, Y., "Learning Hierarchical Features for Scene Labeling," Pattern Analysis and Machine Intelligence, IEEE Transactions on , vol.35, no.8, pp.1915,1929, Aug. 2013

[38]. H. Lee, C. Ekanadham, and A.Y. Ng. Sparse deep belief net model for visual area v2. In NIPS, 2008. 
[39]. G. E. Hinton, S. Osindero, and Y.W. Teh. A fast learning algorithm for deep belief nets. Neural Computation, 18(7):1527-1554, 2006.

[40]. Wright, J.; Yang, A.Y.; Ganesh, A.; Sastry, S.S.; Yi Ma, "Robust Face Recognition via Sparse Representation," Pattern Analysis and Machine Intelligence, IEEE Transactions on, vol.31, no.2, pp.210,227, Feb. 2009

[41]. Elad, M.; Aharon, M., "Image Denoising Via Sparse and Redundant Representations Over Learned Dictionaries," Image Processing, IEEE Transactions on , vol.15, no.12, pp.3736,3745, Dec. 2006

[42]. Donoho, D.L.; Elad, M.; Temlyakov, V.N., "Stable recovery of sparse overcomplete representations in the presence of noise," Information Theory, IEEE Transactions on , vol.52, no.1, pp.6,18, Jan. 2006

[43]. Aharon, M.; Elad, M.; Bruckstein, A., "K-SVD: An Algorithm for Designing Overcomplete Dictionaries for Sparse Representation," Signal Processing, IEEE Transactions on , vol.54, no.11, pp.4311,4322, Nov. 2006

[44]. B. Willmore and D. J. Tolhurst. Characterizing the sparseness of neural codes. Network, 12(3):255-270, January 2001

[45]. A. Treves and E. Rolls. What determines the capacity of autoassociative memories in the brain? Network: Computation in Neural Systems, 2:371-397(27), 1991

[46]. G. E. Hinton, S. Osindero, and Y.W. Teh. A fast learning algorithm for deep belief nets. Neural Computation, 18(7):1527-1554, 2006.

[47]. Y. Bengio, P. Lamblin, D. Popovici, and H. Larochelle. Greedy Layer-Wise training of deep networks. In NIPS, 2006.

[48]. D. J. Field. What is the goal of sensory coding? Neural Computation, 6(4):559-601, July 1994.

[49]. A. Coates, H. Lee, and A. Y. Ng. An analysis of single-layer networks in unsupervised feature learning. In AISTATS, 2011.

[50]. H. Lee, Y. Largman, P. Pham, and A. Y. Ng. Unsupervised feature learning for audio classification using convolutional deep belief networks. In NIPS. 2009.

[51]. Yang Y, Qiu Y, Lu C. Automatic Target Classification-Experiments on the MSTAR SAR Images[C]. Software Engineering, Artificial Intelligence, Networking and Parallel/Distributed Computing, 2005 and First ACIS International Workshop on Self-Assembling Wireless Networks. SNPD/SAWN 2005. Sixth International Conference on. IEEE, 2005: 2-7.

[52]. Boyd S, Parikh N, Chu E, et al. Distributed optimization and statistical learning via the alternating direction method of multipliers[J]. Foundations and Trends in Machine Learning, 2011, 3(1): 1-122. 
[53]. Chambolle, Antonin. "An algorithm for total variation minimization and applications." Journal of Mathematical ima ging and vision 20.1-2 (2004): 89-97.

[54]. Sun Y, Liu Z, Todorovic S, et al. Synthetic aperture radar automatic target recognition using adaptive boosting[C], Defense and Security. International Society for Optics and Photonics, 2005: 282-293. 